\title{
USO DO SISTEMA OTRS PARA GERENCIAR INCIDENTES E ELEVAR O NÍVEL DOS SERVIÇOS OFERECIDOS PELAS EMPRESAS
}

\author{
USE OF THE OTRS SYSTEM TO MANAGE INCIDENTS AND RAISE THE LEVEL OF \\ SERVICES OFFERED BY COMPANIES
}

Tainá Cardoso dos Santos Silva - tainacardoso@outlook.com
Faculdade de Tecnologia (Fatec) - Taquaritinga - SP - Brasil

Gustavo Henrique Del Vechio - gustavo.vechio@fatectq.edu.br Faculdade de Tecnologia (Fatec) - Taquaritinga - SP - Brasil

DOI: 10.31510/infa.v17i2.860

Data de publicação: 18/12/2020

\begin{abstract}
RESUMO
Quanto mais as empresas investirem na interação e automação de processos, mais podem elevar a qualidade de seus serviços e o atendimento ao público. Posto dessa forma, este estudo procura analisar o sistema open source chamado OTRS (Open-source Ticket Request System) e seus recursos para a gestão de incidentes e resolução de problemas no âmbito dos negócios. Após descrever as ferramentas essenciais disponíveis, o estudo realiza uma comparação entre um fluxo de atendimento tradicional e quais as vantagens obtidas ao adotar o OTRS para este processo. O que se nota, a partir deste estudo, é que o OTRS oferece muitas vantagens à tratativa de incidentes, desde um melhor controle e comunicação com clientes até um fluxo mais dinâmico entre diferentes departamentos, a fim de resolver problemas com mais velocidade e eficiência. É, assim, uma escolha muito interessante para empresas que desejam elevar o nível de seus serviços.
\end{abstract}

Palavras-chave: OTRS. ITIL. Gestão de incidentes. Processos automatizados

\begin{abstract}
The more companies invest in the interaction and automation of processes, the more they can increase the quality of their services and attendance to the public. That way, this study seeks to analyze the open source system called OTRS (Open-source Ticket Request System) and its resources for incident management and problem solving in the scope of businesses. After describing the essential tools available, the study makes a comparison between a traditional service flow and what are the advantages obtained by adopting OTRS for this process. What can be seen from this study is that OTRS offers many advantages for handling incidents, from better control and communication with customers to a more dynamic flow between different
\end{abstract}


departments, in order to solve problems more quickly and efficiently. It is, therefore, a very interesting choice for companies that wish to raise the level of their services.

Keywords: OTRS. ITIL. Incident management. Automated processes.

\section{INTRODUÇÃO}

Este estudo tem o objetivo de compreender os principais recursos e as vantagens do sistema open source de Help Desk OTRS, que tem alinhamento direto com as boas práticas do framework ITIL e oferece mais eficiência no atendimento, controle e gerenciamento de incidentes por parte de empresas de diversos portes e segmentos.

Para alcançar este objetivo, a metodologia do estudo se define, primeiramente, pela revisão de materiais especializados, a fim de entender o que é o ITIL e o OTRS, demonstrando, a seguir, os recursos fundamentais deste sistema. Em seguida, para avaliar a pertinência dos conceitos estudados, apresenta-se uma análise cujo propósito é a de comparar o uso e os benefícios do OTRS em relação a um fluxo tradicional de atendimento.

O estudo se justifica por verificar que quanto mais as empresas investirem em recursos dinâmicos e automatizados para a gestão de seus processos, neste caso de incidentes, mais podem elevar sua reputação e seu nível de serviços, o que, certamente, contribui para manter a reputação e a integridade da marca no mercado.

\section{FUNDAMENTAÇÃO TEÓRICA}

O framework ITIL, cuja sigla corresponde a "Information Technology Infrastructure Library", ${ }_{1}^{1}$ representa um conjunto de boas práticas utilizadas no gerenciamento de serviços de TI, cujos benefícios incluem clareza e agilidade na priorização de demandas, redução de incidentes, custos e riscos, nível de serviço dimensionados conforme a necessidade dos setores, processos consistentes e previsíveis, eficiência ne entrega de serviços, além de otimização da experiência do cliente (CORREA, 2018).

Como lembra Chiari (2017), a primeira versão deste framework surgiu em meados de 1980, pela Agência Central de Computação e Telecomunicações do Reino Unido, a CCTA (Central Computer and Telecommunications Agency). Com o tempo, novas versões foram

\footnotetext{
${ }^{1}$ Em tradução livre para o Português, ITIL significa "Biblioteca de Infraestrutura de Tecnologia da Informação".
} 
lançadas para aprimorar seus recursos. O ITIL V2 surgiu entre 2000 e 2001, enquanto o ITIL V3 surgiu em 2007. A última versão lançada é a 4, de fevereiro de 2019, que possui diversos recursos alinhados com as exigências tecnológicas do mercado atual, tais como a criação de valor por meio de serviços de TI (SVS - Service Value System), que considera quatro dimensões do gerenciamento de serviços: (1) pessoas e organizações, (2) informação e tecnologia, (3) parceiros e fornecedores, e (4) cadeia de valor e processos; isso facilita a implementação da estratégia de TI em nuvem, reduzindo riscos desnecessários (CÉSAR, 2019). Além disso, o ITIL 4 traz vantagens no gerenciamento de segurança da informação, gerenciamento de conhecimento e outros aprimoramentos (MOHR, 2019).

\subsection{Sistema OTRS}

Desde que o framework ITIL foi lançado na década de 1980, muitos sistemas foram desenvolvidos com base em sua fundamentação. Um deles é o OTRS (Open-source Ticket Request System), um sistema open source de Help Desk que tem alinhamento direto com as boas práticas do ITIL desde a versão 3, permitindo o atendimento a serviços de forma ampla e eficiente. Embora a versão mais recente do OTRS seja a 8, a última versão gratuita e disponível para a comunidade é a 6.0.28.

O OTRS é amplamente utilizado em empresas de diversos segmentos e tamanhos, já que um dos pontos positivos de sua utilização, de acordo com o próprio fabricante (OTRS, 2020), é a flexibilidade para permitir autonomia na criação e configuração de processos. Esta vantagem encontra-se tanto na interface web, em que os administradores do sistema podem criar processos, serviços, personalizar campos e telas, quanto na parte de desenvolvimento, possibilitando alteração dinâmica de códigos e criação de módulos.

\subsection{Características do sistema OTRS}

Embora este estudo procure demonstrar os benefícios de se usar o OTRS para o gerenciamento de incidentes, a otimização de tempo de atendimento e as facilidades para gerir processos, antes de apresentar tais vantagens é importante conhecer algumas características fundamentais quanto a seus recursos. Tais características são apresentadas nos tópicos a seguir. 


\subsubsection{Gerenciamento de pacotes no OTRS}

O sistema OTRS permite a adição, isto é, a instalação ${ }^{2}$ de pacotes que estendem suas funcionalidades. Conhecidos por módulos ou Add Ons, tais pacotes podem ser desenvolvidos pelo OTRS Group (empresa responsável pelo desenvolvimento do próprio OTRS), ou ainda por terceiros. Por exemplo, na versão 6 do sistema, é possível instalar módulos como gerenciadores de incidentes, de mudanças, contabilização de tempo, dashboards, relatórios de produtividade de agentes, estatísticas personalizáveis, controle de projetos, Kanban, base de conhecimento, controle de SLA (Service Level Agreement), gráficos dinâmicos, integrações com outros sistemas (como Zabbix, OCS Inventory, e WhatsApp), além de outras funcionalidades.

Vale destacar que embora exista a possibilidade de instalar gratuitamente o módulo de gestão de incidentes, ele é pouco utilizado, pois seu fluxo pronto impede alterações. Em vez deste módulo, é mais comum o uso do Gerenciador de Processos (explicado a seguir), capaz de personalizar o fluxo de incidentes.

\subsubsection{Gerenciamento de processos e campos dinâmicos no OTRS}

O Gerenciador de Processos, disponível na aba de Administração do OTRS 6, permite a criação de processos a partir de atividades, janelas de atividades, transições e ações de transições. Conforme descrito pelo Manual do Administrador do OTRS, ${ }^{3}$ essas funcionalidades auxiliam na criação de um fluxograma dentro do sistema, sendo que os campos dinâmicos, além de potencializarem o uso dos recursos, são fundamentais para alterarem as atividades de um processo. Exemplos de campos dinâmicos incluem checkbox, data e hora, menu suspenso, menu de seleção múltipla, input (entrada) de texto curto e input de texto longo. Além destes campos, é possível instalar pacotes adicionais que permitem a criação de campos como tabelas, anexos, controle de tempo contabilizado, entre outros.

Nas configurações do sistema, é possível adicionar estes campos dinâmicos, para que atendentes e clientes possam adicionar informações ao chamado. As atividades dos processos também podem ficar disponíveis a estes usuários, desde que tenham permissão de visualizarem

\footnotetext{
2 Para mais informações sobre a instalação de pacotes no sistema OTRS, pode-se consultar o Manual do Administrador, disponibilizado pelo OTRS Group por meio do link https://bit.ly/3fFoPwB.

${ }^{3}$ No Manual do Administrador do OTRS, a página de Gerenciamento de processos pode ser acessada pelo link https://bit.ly/3daEcvf. Já a página sobre campos dinâmicos pode ser acessada pelo link https://bit.ly/2YIFUi5.
} 
a janela de atividades. Seja como for, todas estas interações são registradas no chamado, podendo ser consultadas a qualquer momento pelo recurso de histórico.

\subsubsection{Gerenciamento de filas e permissões no OTRS}

O Gerenciamento de filas (Queues $)^{4}$ do sistema OTRS, permite a organização das áreas empresariais dentro da ferramenta, de acordo com permissões previamente configuradas. Uma fila é utilizada para separar as áreas da empresa, tais como comercial, financeiro, infraestrutura, desenvolvimento, recursos humanos e projetos. Além disso, as filas segregam permissões por meio de grupos, permitindo ou não determinadas ações para cada tipo de atendente ou cliente. Exemplos de permissões disponíveis incluem:

- Somente leitura: permite que o usuário apenas visualize os chamados e as interações;

- Mover: permite que o usuário mova chamados para a fila;

- Criar: permite que o usuário crie chamados para a fila;

- Nota: permite que o usuário crie notas em chamados;

- Proprietário: permite que o usuário altere o proprietário do chamado;

- Prioridade: permite que o usuário visualize e altere prioridades do chamado;

- Leitura e escrita: concede ao usuário todas as permissões anteriores, além do direito de interagir no chamado, por meio da criação de artigos.

No sistema, estas permissões podem ser atribuídas para os atendentes, por meio de grupos ou papéis. A associação por Atendentes $>$ Grupos permite que as permissões sejam adicionadas aos analistas, enquanto a associação por Atendentes $>$ Papéis permite que as permissões sejam adicionadas por equipes. Em organizações de médio ou grande porte, o ideal é que a associação seja feita por Atendentes $>$ Papéis, pois isso evita conceder acessos incorretos para cada atendente (como seria em Atendentes $>$ Grupos). O grupo que foi associado ao usuário cliente ou ao atendente é utilizado na criação da fila, permitindo que os atendentes visualizem e trabalhem nos chamados que possuem permissão, enquanto os usuários clientes podem abrir e visualizar chamados para as filas que têm permissão.

\footnotetext{
${ }^{4}$ Mais informações sobre o Gerenciamento de Filas do OTRS 6 encontram-se no Manual do Administrador do OTRS, em https://bit.ly/2Ndvdi5. Já as políticas para grupos estão em https://bit.ly/30QgK3I.
} 


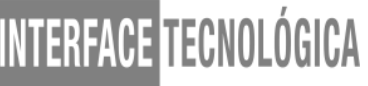

\subsection{Gerenciamento de serviços e acordo de nível de serviço}

No sistema OTRS, clientes podem ser associados a um serviço, tendo a possibilidade de vinculação por usuário ou, ainda, a associação de serviços padrões para todos os usuários. ${ }^{5}$ Além disso, é possível associar um cliente a um serviço. A diferença entre usuários clientes e clientes é que os primeiros utilizam o produto ou serviço (pessoas reais), enquanto os segundos são responsáveis por adquirir os produtos e serviços (empresas).

Os serviços são utilizados para classificar as atividades de um chamado. Por exemplo, uma empresa provedora de Internet pode abrir um novo chamado quando percebe indisponibilidade de conexão; para isso, deve saber o tipo de serviço a ser incluído no sistema. Todo serviço, por conseguinte, possui um prazo de atualização, primeira resposta e solução (no sistema, esse fluxo é chamado de SLA): enquanto o prazo de atualização é o intervalo de tempo que os analistas interagem com o usuário cliente, o prazo de primeira resposta refere-se ao tempo que os analistas adicionam a primeira resposta no chamado. Já o prazo de solução é o tempo em que o analista tem para solucionar e, logo em seguida, fechar e chamado.

Por meio das configurações do sistema, também é possível acrescentar calendários, a fim de planejar o trabalho semanal (que pode ou não incluir feriados). Aliás, por meio da instalação de um módulo adicional chamado "Znuny4OTRS-EscalationSuspend", é possível pausar a contabilização dos prazos de SLA nos estados configurados, classificando-os como pendentes (por exemplo, pendente com o cliente e aguardando validação).

\subsection{Gerenciamento de estados no OTRS}

Os estados ${ }^{6}$ são utilizados para sinalizar em qual parte do "ciclo de vida do chamado", um chamado encontra-se. Mais especificamente, quando um chamado é criado, recebe o estado de "novo"; caso um atendente ou usuário cliente interaja no chamado, o estado passa para “aberto". Após o início do atendimento, o estado pode ser alterado para "em atendimento"; quando é enviado para validação do usuário cliente, torna-se "pendente com o cliente" e, após ser resolvido com sucesso, atinge o estado de "fechado com êxito".

\footnotetext{
${ }^{5}$ No Manual do Administrador do OTRS, os níveis de serviços podem ser consultados em https://bit.ly/3fuqSDi.

${ }^{6}$ No Manual do Administrador do OTRS, a descrição de estados pode ser consultada em https://bit.ly/2YKgaSw.
} 
Esses são apenas alguns exemplos de estado utilizados, uma vez que novos estados podem ser criados de acordo com a necessidade da empresa. Embora na criação de estados seja possível definir um nome personalizado, é preciso informar qual é o seu tipo: aberto, agrupado, fechado, lembrete de pendente, novo, pendente automático e removido.

\subsection{Atendentes no OTRS}

O OTRS permite que os chamados tenham proprietários e responsáveis. ${ }^{7}$ Os proprietários são aqueles que executam as atividades das partes do processo, enquanto os responsáveis são os que tem responsabilidade no chamado e interagem com atendentes e usuários clientes. Um exemplo desse fluxo de trabalho está nos incidentes que envolvem falhas (bugs): o responsável precisa mover o chamado para a fila de desenvolvimento, a fim de que um desenvolvedor receba o papel de proprietário do chamado. Este desenvolvedor não tem contato direto com o cliente, ficando a cargo do responsável realizar a comunicação.

\section{PROCEDIMENTOS METODOLÓGICOS}

Entendido os conceitos fundamentais para se utilizar o sistema OTRS, apresenta-se, a seguir, uma análise a fim de verificar como é realizado um atendimento feito por telefone (a seguir denominado “tradicional"), em comparação com a adoção do OTRS. Neste caso, a versão do sistema utilizada é a 6 , pois trata-se da última versão gratuita e disponível à comunidade.

Deve-se lembrar que como o OTRS é flexível e personalizável via código e interface, ele pode ser utilizado em diversos segmentos ou empresas. Por exemplo, uma empresa de agropecuária pode adotar o OTRS para gerenciar os setores e atendimentos, enquanto uma empresa de engenharia, uma fábrica alimentícia ou um grupo de TI podem fazer o mesmo.

É esperado que o gerenciamento de incidentes seja realizado pelo departamento de TI da empresa. Em um fluxo tradicional, o cliente geralmente liga para a Central de Atendimento, informa um número de autenticação, digita o ramal que deseja solicitar atendimento e aguarda um analista ficar disponível para ajudá-lo, o que pode gerar um certo desgaste por parte do cliente, caso tenha que esperar muito tempo para ser atendido.

\footnotetext{
${ }^{7}$ Mais informações sobre proprietários e responsáveis podem ser consultadas em: https://bit.ly/316X9g7.
} 
Com o sistema OTRS, este processo ocorre de maneira mais facilitada, sobretudo porque o cliente não precisa perder tempo ao telefone para registrar um incidente. Basta, neste caso, fazer login no portal disponível na $w e b,{ }^{8}$ selecionar o serviço que deseja atendimento e preencher campos como assunto e texto de comentário; somente esses passos são suficientes para abrir um novo chamado e notificar a equipe responsável pelo serviço.

Outra vantagem deste fluxo com o OTRS é que o chamado pode entrar no sistema com um direcionamento à fila correta de serviços, além de já carregar informações tais como prioridade e tipo de serviço. Com isso, evita-se a necessidade de um analista perder tempo em categorizar esse chamado ou se preocupar com tais questões; isso dá a ele mais tempo para focar no que realmente importa, que é solucionar o problema (aliás, se o analista precisar escalar o incidente a outro profissional ou superior, no OTRS isso pode ser feito facilmente com poucos cliques). Já em um fluxo tradicional, este processo seria mais burocrático, já que os analistas geralmente deveriam preencher manualmente um formulário e enviar ao cliente para validar as informações, muitas vezes sem ter um padrão no catálogo de serviços ou controle de prioridades. Vale destacar que, no OTRS, após abrir um chamado, o cliente recebe automaticamente a confirmação de abertura via e-mail, contendo o número do atendimento. Além disso, também é importante salientar que a abertura de chamado no OTRS é instantânea, o que facilita a tratativa de incidentes graves (em um fluxo tradicional, a comunicação seria realizada por telefone e e-mail, para que depois o chamado começasse a ser atendido).

Mais um aspecto importante do OTRS é que ele conta com uma base de conhecimento que é capaz de restringir grupos de visualização, além de permitir a associação de procedimentos específicos a cada chamado adicionado à fila. Esta também é uma vantagem em comparação a um fluxo tradicional, que pode precisar utilizar documentos compartilhados online a fim de facilitar o controle de incidentes e o trabalho dos coordenadores.

Caso a empresa opte por oferecer ao usuário opções de procedimentos antes da abertura do chamado, basta associar um identificador de questões frequentes $(F A Q)$ ao serviço. Neste caso, quando o cliente estiver na tela para abrir um chamado, uma ou mais sugestões de procedimentos são automaticamente exibidas. Se, após realizar estes procedimentos comuns, o problema ainda persista, o cliente pode optar por abrir, de fato, o chamado. Essa é uma funcionalidade prática e bastante flexível do OTRS, que garante mais agilidade nas tratativas e

\footnotetext{
${ }^{8}$ A interface web pode ser criada e gerenciada por um módulo do OTRS chamado InBox Customer Portal. Para mais informações, pode-se acessar o link https://bit.ly/3dyqLWg.
} 
menor demanda de trabalho para os atendentes, já que, em muitos casos, o próprio cliente acaba encontrando uma solução útil ao problema. Embora este conceito possa ser aplicado a fluxos tradicionais de atendimento, tal opção mostra-se mais difícil pois as gravações telefônicas dos problemas mais comuns são limitadas e de acesso mais complicado.

Com um chamado registrado na fila por parte do cliente, é possível, por meio do OTRS, acompanhar o ciclo de evolução até a solução do incidente, em seguida criando, até mesmo, estatísticas que incluem a quantidade de chamados em análise, em execução, em validação e até os chamados fechados. Isso possibilita um controle eficiente de cada chamado, além de oferecer análises de desempenho de cada analista. Os gestores podem, neste sentido, ter acesso a relatórios em tempo real, o que proporciona uma visão muito ampla do serviço (estes também são quesitos mais difíceis de serem controlados em um fluxo tradicional de atendimento).

No OTRS, diferente de um atendimento tradicional, o cliente recebe automaticamente por e-mail a informação de que o analista finalizou as atividades na tratativa de seu chamado. Neste caso, o cliente tem alguns dias para responder o e-mail confirmando que o incidente foi resolvido; caso não o faça, o sistema automaticamente encerra o chamado.

Com o chamado encerrado, o próprio sistema OTRS envia, por e-mail, uma pesquisa de satisfação ao cliente; neste caso, coordenadores e gestores podem analisar as respostas e gerar estatísticas inerentes aos atendimentos diários, semanais ou mensais. Estes e outros recursos do OTRS demonstram que ele possui uma unificação em termos de comunicação; em outras palavras, todas as interações com os clientes permanecem em uma única base de dados, facilitando consideravelmente a gestão e o acesso sempre que for necessário.

\section{RESULTADOS E DISCUSSÃO}

Como é possível verificar a partir das análises comparativas entre um fluxo tradicional de atendimento e a adoção do sistema OTRS, há diversas vantagens quanto ao uso do OTRS, tanto para os clientes abrirem novos chamados e acompanharem a solução de incidentes, quanto para as empresas e seus departamentos de TI tentarem solucionar casos com o máximo possível de eficiência (inclui-se, aqui, o fato do sistema ser totalmente personalizável às demandas de cada negócio). Destaca-se, neste sentido, o controle de SLA (Service Level Agreement), que permite que os administradores registrem o tempo de primeira resposta, atualização e solução de serviços, sem que o analista tenha que categorizar manualmente o chamado, como ocorreria 


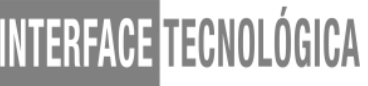

em um atendimento tradicional. Aliás, o gerenciamento de processos do OTRS também proporciona uma dinâmica mais eficiente para os analistas interagirem, registrarem e executarem atividades, sem correr o risco de esquecerem etapas no fluxo do trabalho.

Outro ponto interessante está nos relatórios gerados pelo OTRS, que são essenciais para os gestores extraírem informações de atendimento e, com isso, medir a produtividade dos profissionais responsáveis e dos chamados em geral (não há, portanto, a necessidade de os analistas ou coordenadores gerarem manualmente relatórios). Além disso, o recurso de pesquisa de satisfação, também disponível no sistema, oferece diversos dados bastante relevantes a administradores, a fim de melhorar cada vez o nível de seus serviços. Todos esses dados, vale destacar, permanecem no dashboard, permitindo uma visão muito ampla de cada processo, recurso ou informação coletada. É, portanto, um centro de controle com recursos de fácil acesso e que potencializa o trabalho, em comparação a um fluxo tradicional de atendimento.

Destaca-se, ainda, o fato de os próprios clientes serem capazes de acompanhar todo o fluxo de atendimento para os chamados que fazem, algo dificilmente obtido em um fluxo tradicional de processos. Neste caso, os clientes podem não apenas verificar, mas interagirem com seus chamados, a fim de manter uma comunicação mais estreita com os analistas responsáveis. Toda essa interatividade, vale lembrar, permanece no histórico de cada chamado e pode ser configurada para envio ao cliente via e-mail; assim, mesmo que ele não acompanhe diretamente pelo sistema, pode acompanhar seus chamados sem maiores dificuldades.

Esses, afinal, são aspectos que demonstram o quanto um sistema interativo como o OTRS, que é open source e possui versão gratuita, pode ajudar a potencializar o atendimento e o nível de serviços prestados por empresas dos mais diferentes portes e ramos de atuação.

\section{CONSIDERAÇÕES FINAIS}

Este estudo procurou demonstrar como o uso do sistema open source OTRS pode auxiliar no gerenciamento de incidentes, seguindo as boas práticas definidas pelo popular framework ITIL. Como pode ser verificado pelas análises e comparações, os principais recursos deste sistema incluem a flexibilidade para a configuração e o gerenciamento de casos, a centralização das informações em uma única base, a geração de relatórios personalizados, além da possibilidade de instalação de módulos adicionais que garantem mais personalização, de acordo com as regras de cada negócio ou o suporte às diferentes áreas de uma empresa. 


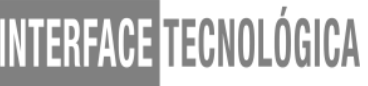

Aliás, pela comparação do OTRS com um fluxo tradicional de atendimento, é possível avaliar que as atividades, as interações e os registros de chamados do OTRS melhoram o fluxo de gestão de incidentes para coordenadores, gestores, clientes e principalmente atendentes, já que várias atividades podem ser automatizadas, liberando mais tempo para que os profissionais se dediquem no que realmente importa, que é encontrar soluções para os problemas. O OTRS demonstra, assim, diversas vantagens no registro, tratativa, finalização e gestão de chamados sendo, portanto, uma escolha muito interessante para quaisquer empresas que desejam elevar seu nível de serviços e ter mais eficiência na resolução de problemas.

\section{REFERÊNCIAS}

CHIARI, Renê. O que é ITIL? Tudo o que você precisa saber sobre o tema. ITSM na Prática, 2017. Disponível em: < https://bit.ly/30T4RKG >. Acesso em: 17 jun. 2020.

CÉSAR, Flávio. O que podemos ganhar com ITIL v4? Profissionais TI, 14 maio 2019. Disponível em: < https://bit.ly/3eqQ4uo $>$. Acesso em: 23 jun. 2020.

CORREA, Rafael M. ITIL 4: confira o que mudou no framework e descubra como criar valor

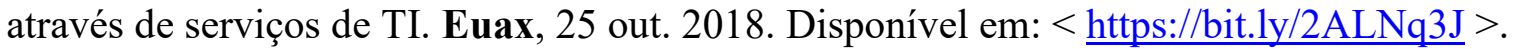
Acesso em: 17 jun. 2020.

MOHR, Julie L. ITIL 4 and Artificial Intelligente White Paper. Axelos, fev. 2019. Disponível

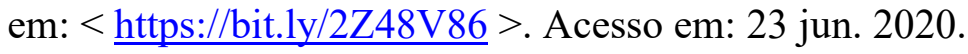

OTRS. Soluções que se encaixam perfeitamente. OTRS, 2020. Disponível em: < https://bit.ly/2AOED15 >. Acesso em: 18 jun. 2020.

OTRS GROUP. Manual do Administrador do OTRS. OTRS Group, s.d. Disponível em: < https://bit.ly/3fBfp4Z >. Acesso em: 18 jun. 2020. 1993. 